\title{
Adipose-Derived Mesenchymal Stem Cells Attenuate Acute Lung Injury Through Regulation of the Gut Microbiota in Septic Rats
}

\section{Junyi Sun}

Zhengzhou University First Affiliated Hospital

\section{Xianfei Ding}

Zhengzhou University First Affiliated Hospital

Shaohua Liu

Zhengzhou University First Affiliated Hospital

\section{Xiaoguang Duan}

Zhengzhou University First Affiliated Hospital

\section{Huoyan Liang}

Zhengzhou University First Affiliated Hospital

tongwen sun ( $\square$ suntongwen@163.com )

Zhengzhou University First Affiliated Hospital https://orcid.org/0000-0001-6536-3222

\section{Research}

Keywords: Acute lung injury, mesenchymal stem cells, sepsis, cecal ligation and puncture, gut microbiota

Posted Date: May 21st, 2020

DOI: https://doi.org/10.21203/rs.3.rs-29551/v1

License: (9) This work is licensed under a Creative Commons Attribution 4.0 International License. Read Full License

Version of Record: A version of this preprint was published on September 7th, 2020. See the published version at https://doi.org/10.1186/s13287-020-01902-5. 


\section{Abstract \\ Background}

We hypothesized that adipose-derived mesenchymal stem cells (ADMSCs) may ameliorate sepsisinduced acute lung injury (ALI) by increasing or decreasing microorganism populations in the gut microbiota, such as that of Firmicutes and Bacteroidetes.

\section{Methods}

A total of 60 male adult Sprague-Dawley (SD) rats were separated into three groups: the sham control (SC) group, the sepsis induced by cecal ligation and puncture (CLP) group, and the ADMSC treatment (CLP-ADMSCs) group, in which rats underwent the CLP procedure and then received $1 \times 10^{6} \mathrm{ADMSCs}$. Rats were sacrificed 24 hours after the SC or CLP procedures. To investigate the relationship between sepsis-induced ALI and the gut microbiota, rat lungs were histologically evaluated using hematoxylin and eosin (H\&E) staining, serum levels of pro-inflammatory factors were detected using enzyme-linked immunosorbent assay (ELISA), and fecal samples were collected and analyzed using 16S rDNA sequencing.

\section{Results}

The serum levels of inflammatory cytokines, tumor necrosis factor (TNF)- $a$ and interleukin (IL)-6, were significantly increased in rats after the CLP procedure, but were significantly decreased in rats treated with ADMSCs. Histological evaluation of the rat lungs yielded results consistent with the changes in IL-6 levels among all groups. Treatment with ADMSCs significantly increased the diversity of the gut microbiota in rats with sepsis. The principal coordinates analysis (PCOA) results showed that there was a significant difference between the gut microbiota of the CLP-ADMSCs group and that of the CLP group. In rats with sepsis, the proportion of Bacteroides related to energy consumption and Escherichia-Shigella related to lipopolysaccharide production increased, and the proportion of Akkermansia related to the regulation of intestinal mucosal thickness and the maintenance of intestinal barrier function decreased. Furthermore, the proportion of Firmicutes related to energy storage, such as Verrucomicrobia, decreased. Intervention with ADMSCs increased the proportion of beneficial bacteria, reduced the proportion of harmful bacteria, and normalized the gut microbiota, thus, improving the sepsis-induced ALI.

\section{Conclusions}

Therapeutically administered ADMSCs may improve CLP-induced ALI by regulating the gut microbiota, providing a potential mechanism by which mesenchymal stem cells treat sepsis. 


\section{Background}

Sepsis is a public health problem characterized by life-threatening organ dysfunction due to maladjustment of the host response to infection ${ }^{[1,2]}$. In the intensive care unit (ICU), the global mortality rate of sepsis is close to $25 \%^{[3,4]}$. Sepsis involves the damage of various important organ functions, including brain, kidney, and heart functions ${ }^{[5-8]}$. ALI can easily occur in the early stage of sepsis due to the involvement of the lungs in suppurative inflammation ${ }^{[9]}$. And there are many potential pathogens that can cause sepsis ${ }^{[10]}$. More importantly, there is no drug or treatment that can effectively cure sepsis ${ }^{[11]}$. Therefore, it is necessary for us to develop new therapeutic methods for ALI treatment.

In recent years, ADMSCs have been used as a new treatment strategy for diseases related to inflammation and tissue damage ${ }^{[12-15]}$. ADMSCs can alleviate inflammation and oxidative stress ${ }^{[16,17]}$, and many studies have confirmed the therapeutic effect of mesenchymal stem cells (MSCs) in sepsis animal models ${ }^{[18-20]}$. Recent studies have found that ADMSCs and cord-derived MSCs show therapeutic effects on inflammation and maintain the balance of the gut microbiota after early cell injection ${ }^{[21]}$.

The gut microbiota is a complex microbial community that has been shown to play a major role in health and disease ${ }^{[23]}$. The gut microbiota consists of microorganisms that reside in the host gastrointestinal tract. These microorganisms provide protection against pathogen colonization and invasion through appropriate responses, leading to inflammation or tolerance. Humans have nearly 100 trillion gut bacteria that are vital for good health. Millions of years of coevolution have shaped the symbiotic relationship between humans and microorganisms, in which the gut bacteria play a crucial role in the metabolism of human nutrients, while the human gut provides a nutrient rich environment for the bacteria. Therefore, an imbalance in the gut microecology leads to the occurrence or aggravation of disease ${ }^{[24-29]}$. Dysfunction of the intestinal epithelium, dysfunction of the immune system, and translocation of enteropathogenic bacteria are considered to be the key factors leading to infectious complications and multiple organ dysfunction syndromes. The gut is considered to be a driving factor for sepsis and multiple organ dysfunction syndromes ${ }^{[30]}$. As most of the microorganisms in the human intestine are anaerobic, once excreted with feces, their biological activity is lost ${ }^{[31]}$. Therefore, the composition of the intestinal microflora detected by clinical fecal samples through bacterial culture does not fully reflect the overall picture of the human intestinal microecology. To address this problem, in animal experiments, feces collected directly from the intestine can be used for microbiological analysis to better reflect the overall picture of the intestinal microecology. 16S rDNA sequencing is a method for high-throughput and rapid identification of bacterial species ${ }^{[32]}$. In this study, we used 16S rDNA technology to observe the effect of early treatment with ADMSCs on the intestinal microecology of rats with sepsis, and we compared the general changes in intestinal bacteria between the different treatment groups.

In this study, SD rats with sepsis induced by CLP were used to test whether treatment with ADMSCs could improve the ALI caused by sepsis through changes in the intestinal microbiome. 


\section{Methods}

Experimental materials

Animals

A total of 60 male adult SD rats (200-250 g, 6-8 weeks old) were purchased from Charles River (Beijing, China, http://www.vitalriver.com) and placed in the temperature control and light control room $\left(20-25^{\circ} \mathrm{C}\right.$, 1:1 light dark cycle) for free feeding and drinking. Rats were randomly divided into three groups (20 rats in each group): the SC group, the CLP group, and the CLP-ADMSCs treatment group. The SC and CLP groups were injected with normal saline, and the CLP-ADMSCs group was injected with $1 \times 10^{6} \mathrm{ADMSCs}$ through the tail vein 1 hour after CLP operation. Rats were killed 24 hours after the operation and their serum, feces, and lung tissue samples were collected for analysis. This study was conducted in accordance with the principles described in the National Institutes of Health $(\mathrm{NIH})$ guidelines for the care and use of laboratory animals (http://grants1.nih.gov/grants/olaw/). All experiments were approved by the ethics review committee of life sciences at Zhengzhou University.

Main reagents

$10 \%$ chloral hydrate was purchased from SLB-bio (Beijing, China). ADMSCs were purchased from Cyagen (China). ELISA kits of rat IL- 6 and TNF-a were purchased from R\&D Systems (USA). $0.25 \%$ ethylenediaminetetraacetic acid (EDTA) trypsin was purchased from SLB-bio. Phosphate buffered saline (PBS) and the E.Z.N.A. ${ }^{\circledR}$ Stool DNA Kit were purchased from HyClone Laboratories (USA) and Omega Biotek (USA), respectively. The Library Quantification Kit for Illumina ${ }^{\circledR}$ was purchased from Kapa Biosciences (Woburn, USA). Fetal bovine serum and penicillin mixture $(100 \times)$ were purchased from Gemini Bio (USA) and SLB-bio, respectively. Dimethyl sulfoxide (DMSO) and isopropyl alcohol were purchased from Sigma (USA) and Tianjin Hengxing Chemical Reagent Manufacturing Co., Ltd. (Tianjin, China), respectively.

Main instruments

The ultra-clean workbench and carbon dioxide incubator used in this study were produced by Thermo Fisher Scientific (USA). The water bath was manufactured by Zhongkelianyi Technology (Beijing, China). The manufacturers of the optical microscopes and micro-samplers were Olympus (Japan) and Eppendorf (USA), respectively. The cell culture plates, cell cryopreservation tubes, and culture flasks were produced by Corning (USA). The 2100 Bioanalyzer system was manufactured by Agilent (Santa Clara, USA). The manufacturers of the $-20^{\circ} \mathrm{C}$ and $-80^{\circ} \mathrm{C}$ refrigerators were Haier (China) and Thermo Fisher Scientific, respectively.

Methods

CLP-induced sepsis rat model 
The CLP model was simulated based on previous literature ${ }^{[33]}$. Briefly, after intra-peritoneal injection of $10 \%$ chloral hydrate $(350 \mathrm{mg} / \mathrm{kg}$ ), the rat abdomen was scraped and thoroughly cleaned with compound iodine, and surgery was performed on a sterile plate. A $2.0 \mathrm{~cm}$ incision was made at the midline of the abdomen. The cecum was ligated and punctured twice with a $10 \mathrm{~mL}$ syringe needle at the blind end, and a certain quantity of feces was squeezed out and placed in the peritoneal cavity. The abdominal muscle layer was sutured with sterile $5+0$ surgical sutures, and the skin was sutured with sterile $3+0$ surgical sutures. All rats were immediately placed in a warm environment after surgery and subcutaneously injected with pre-heated normal saline $(50 \mathrm{~mL} / \mathrm{kg})$ for fluid resuscitation ${ }^{[34]}$. Rats in the SC group were treated similarly, but the cecum was not ligated or punctured.

ADMSC culture and labeling

Second-generation ADMSCs extracted from rat groin fat were purchased from Cyagen Biotechnology (Yangzhou, China, http://www.cyagen.com/cn/zh-cn/). Cells were cultured according to the manufacturer's instructions. Flow cytometry analysis showed that CD29, CD90, and CD44 were positively expressed, while CD 45, CD11b, and CD34 were negatively expressed in the ADMSCs. The cells were cultured in medium $(25 \mu \mathrm{mol} / \mathrm{L})$ to the fourth passage and maintained for 24 hours for later use.

\section{ELISA analysis}

Blood samples were collected from the abdominal aorta of the rats. After 30 minutes, serum was collected and centrifuged ( $2500 \mathrm{rpm}, 15$ minutes). Serum samples were stored at $-80^{\circ} \mathrm{C}$ for ELISA analysis.

Histological analysis of rat lungs

Rat chests were opened to expose the heart, the inferior vena cava was cut off, and a perfusion needle was inserted into the apex of the heart. $100-200 \mathrm{~mL}$ of cold saline was rapidly pushed into the left ventricle and aorta. When the lung turned white, half of the lung tissue was extracted and put into $4 \%$ paraformaldehyde for histological analysis, and the other half was rapidly frozen in liquid nitrogen. The lung tissue was embedded in paraffin and sliced using a slicer. H\&E staining was used to evaluate the lung tissue from the rats. The procedure has been described in detail in the related literature ${ }^{[35]}$. The lung tissue was resected at a size of $5 \mu \mathrm{m}$ and sectioned. H\&E staining was used to analyze the lung tissue.

Sequencing and analysis of the gut microbiota

24 hours after the operation, the rat feces from each group was squeezed into $2 \mathrm{~mL}$ cryopreservation tubes, labeled uniformly, and stored at $-80^{\circ} \mathrm{C}$. The $16 \mathrm{~S}$ rDNA was sequenced using a NovaSeq PE250 platform (Illumina) after the bacterial DNA was extracted from the rat intestine (Hangzhou Lianchuan Biotechnology Co., Ltd., Hangzhou, China). In order to ensure the efficiency and quality of the DNA extracted from the different microorganism sample sources, the best DNA extraction method for the microorganism group was selected and the quality of the extracted DNA was detected using agarose gel 
electrophoresis. Meanwhile, DNA was quantified using an ultraviolet spectrophotometer. The electrophoresis detection time of the polymerase chain reaction (PCR) products was generally within 48 hours, and the bands were irregular or even disappeared after 48 hours. LC-bio uses AMPure XT beads (Beckman Coulter Genomics, Danvers, MA, USA) to purify PCR products and Qubit (Invitrogen, USA) for quantification. The size and quantity of the amplification library were evaluated using the library quantitative kit of the 2100 Bioanalyzer and Illumina (Kapa Biosciences, Woburn, MA, USA), respectively. Alpha diversity was used to analyze the complexity of the sample species diversity. The five indicators, including Chao1, Observed species, Goods coverage, Shannon, and Simpson, in the samples, were calculated using the QIIME2 microbiome bioinformatics platform (https://qiime2.org). Other diagrams were implemented using R-package (v3.5.2) ${ }^{[36-38]}$. 16S rDNA high-throughput sequencing technology was used to analyze the intestinal microecological diversity, as well as to identify and compare the differences in the composition of the gut microbiota from the different treatment groups.

\section{Statistical analysis}

All results were expressed as the mean \pm standard error. One-way analysis of variance (ANOVA) and Tukey's post hoc test were used to evaluate the differences among the three groups. The difference was statistically significant when $P<0.05$. We used SPSS Statistics 21.0 (IBM, Chicago, IL) software to analyze the data.

\section{Results}

ADMSCs can improve the 24-hour survival rate of rats, reduce lung injury, and reduce TNF- $a$ and IL-6 serum levels

The cecum of CLP-induced sepsis rats was congested and necrotic, while the cecum of rats from the CLP-ADMSCs group was significantly improved (Figure S1, macroscopic view of the rat model). In addition, the 24-hour mortality rates of the SC group, CLP group, and CLP-ADMSCs group were $0 \%(0 / 20)$, $40.0 \%(8 / 20)$, and $25.0 \%(5 / 20)$, respectively. The 24-hour mortality rate of the CLP group was

significantly higher than that of the SC group $(P=0.003)$ and the CLP-ADMSCs group $(P=0.501)$. The 24hour mortality rates and $P$ values for each experimental group are shown in Table 1.

Table 1

24-hour mortality of animals in each group.

\begin{tabular}{|lll|}
\hline Group & Fatality rate (deaths / total) & $P$ value \\
\hline SC & $0 \%(0 / 20)$ & $0.003^{\mathrm{a}}$ \\
\hline CLP & $40.0 \%(8 / 20)$ & $0.501^{\mathrm{b}}$ \\
\hline CLP-ADMSCs & $25.0 \%(5 / 20)$ & \\
\hline a, Compared with CLP group;b, Compared with CLP-ADMSCs group \\
\hline
\end{tabular}


ELISA was used to detect the levels of IL-6 and TNF-a in 24-hour serum. The results showed that the serum levels of IL-6 $(P<0.001)$ and TNF-a $(P<0.01)$ were significantly increased, and treatment with ADMSCs significantly reduced the levels of IL-6 $(P<0.01)$ and TNF-a $(P<0.05)$, as shown in Fig. 1 (A\&B).

As shown in Fig. 1(C), H\&E staining revealed increased inflammatory infiltration, edema, and hemorrhage in the CLP group; and, treatment with ADMSCs improved these abnormal pathological manifestations.

Alpha diversity of the gut microbiota in rats

The abundance curve shown in Fig. 2 was used to evaluate whether the detected 16S rDNA sequences covered all microbial species in the sample. The abscissa in the graph represents the number of randomly selected sequences, and the ordinate represents the number of observed operational taxonomic units (OTUs). A short sample curve indicated a small number of sequences. A plateau in the curve indicated that the sequencing was saturated and increasing the amount of data would not obtain new OTUs; however, if the curve did not plateau, the sequencing was not saturated and increasing the amount of data would yield more OTUs. It can be seen from Fig. 2 that there is a turning point in the richness curve of each sample when the sequence number was about 3000 , and that the Shannon curve tends to be flat. This proves that the amount of sequencing data from each group of samples was saturated.

Beta diversity of the gut microbiota in rats

Beta diversity refers to the species diversity among different environmental communities. Together, beta diversity and alpha diversity constitute the overall diversity or the biological heterogeneity of a certain environmental community. To observe differences between samples, beta diversity analysis usually starts with the calculation of the distance matrix between environmental samples, which includes the distance between any two samples, mainly through principal component analysis (PCA), principal coordinates analysis (PCoA), clustering analysis (UPGMA), multidimensional scaling (NMDS), analysis of similarities (ANOSIM), permutational multivariate ANOVA (PERMANOVA, also known as Adonis), and other methods.

PCoA analysis is a visual method to study the similarities or differences between data. PCoA can also be used to observe the differences between individuals or groups. In the two-dimensional scatter diagram of the PCoA in Fig. 3(A), the different groups are represented by different colors. The distance between the samples represents the similarity between the samples, where samples that are closer together are more similar in microbial composition. In the scatter plot, rat samples from the same group gathered together, indicating that the similarity between the gut microbiota of rats from the same group was very high. The greater the distance between different rat groups, the lower the similarity between the samples. In Fig. 3, the degree of contribution by pcoa 1 and pcoa 2 is 0.3585 and 0.1063 , respectively, which can be used to distinguish the intestinal microflora of the rats from the different treatment groups. Therefore, based on the unweighted PCoA in Fig. 3(A), the CLP group was significantly different from the control group and, after ADMSC treatment, the overall similarity between the SC group and the CLP-ADMSCs group was restored. In addition, the PCA in Fig. 3(B) was consistent with the PCoA results, indicating that ADMSCs had a positive impact on the number and diversity of intestinal microorganisms. The PCA showed that 
the structure of the gut microbiota changed significantly after CLP treatment compared to the SC group, but there was little difference between ADMSC-treated rats and the SC group.

Structure analysis of the gut microbiota in rats

In order to further explore the effect of treatment with ADMSCs on the composition of the rat gut microbiota, we statistically analyzed the phylum and genus of the microorganisms in the gut flora. Figure 4(A) shows the distribution of Bacteroidetes, Firmicutes, Verrucomicrobia, and Proteobacteria. Compared to the CLP Group, the abundance of Proteobacteria and Bacteroidetes decreased, and the proportion of Verrucomicrobia increased in the SC group, indicating that CLP had a great influence on the composition of the intestinal microflora. On the contrary, treatment with ADMSCs reduced the intestinal disturbance and the abundance of Proteobacteria. Figure 4(B) shows the genus distribution. Under normal conditions, the abundance of Escherichia-Shigella was very low, while that of the CLP Group was significantly increased. The production of lipopolysaccharide (LPS) by Escherichia-Shigella destroys the balance of the gut microbiota ${ }^{[39-41]}$. The abundance of Bacteroidetes in the SC group was the highest, but that in the CLP group was significantly lower. Figure 4(C) shows that treatment with ADMSCs increased the abundance of Akkermansia, which can regulate the thickness of the intestinal mucosa, so as to maintain the intestinal barrier and provide a protective effect ${ }^{[42-44]}$. The results showed that treatment with ADMSCs could regulate and improve the microflora of rats with sepsis, and had a tendency to restore its abundance. Figure 4(D) shows that the bacterial abundance of the CLP-ADMSCs group is close to that of the SC group, and that there is no significant difference or the difference is smaller than that of the SC group. Overall, the above results suggest that sepsis causes structural changes to the gut microbiota in rats.

\section{Discussion}

This study shows that the gut microbiota of rats is significantly disturbed after sepsis, and that the imbalance of the intestinal ecosystem plays an important role in the development of sepsis-induced ALI. Furthermore, treatment with ADMSCs can alleviate or even restore its normal microbial state and improve ALI. This finding suggests that the potential mechanism by which ADMSCs treat sepsis-induced ALI in rats involves the regulation of the gut microbiota.

The purpose of this study was to determine whether ADMSC treatment could improve ALI in rats with sepsis through alterations in the gut microbiota. At present, more and more bone marrow-derived stem cells are used to treat diseases related to inflammation and organ damage ${ }^{[45]}$. However, little is known about the contribution of ADMSCs to the development of ALI in rats with sepsis ${ }^{[46]}$. Therefore, in order to fill this knowledge gap, in this study, we analyzed changes in the intestinal microflora of sepsis-induced SD rats using $16 \mathrm{~S}$ rDNA sequencing. Through the systematic analysis of human fecal samples, it has been reported that the characteristics of the intestinal microflora are completely changed during sepsis. In the case of severe sepsis, there are serious disorders of the intestinal microenvironment and microflora composition. Since most of the microorganisms in the human intestinal tract are anaerobes, once 
excreted with feces, these microorganisms lose their biological activity. Therefore, it is unnecessary to sequence the feces of patients. To accurately reflect the gut microbiota of patients, this study uses an animal experiment, in which feces is taken from the intestine for flora analysis ${ }^{[47,48]}$.

In order to study the role of the gut microbiota in rats with sepsis, our research team used 16S rDNA analysis technology for the first time to evaluate changes in the gut microbiota of rats after treatment with ADMSCs in the early stage of ALI caused by sepsis. The histological staining results showed that the lungs of rats with sepsis had serious lung injury, and the 16S rDNA sequencing results showed the characteristics of the gut microbiota disorder in the rats. Intervention with ADMSCs reduced organ damage, as well as inflammatory infiltration and hemorrhage, in the lungs, and the diversity of the flora and the content of pathogenic bacteria in rats with sepsis increased significantly. In addition, we found that treatment with ADMSCs also reduced the expression of pro-inflammatory factors, such as TNF- $a$ and IL-6, which is consistent with our previous studies ${ }^{[49,50]}$.

Firmicutes and Bacteroidetes are the main microflora in the human intestinal tract, and they are also the two most abundant microflora in the rat intestinal tract. Firmicutes and Bacteroidetes mainly participate in the regulation of fat and bile acid metabolism and the maintenance of the energy balance in the host ${ }^{[51]}$. Firmicutes help the body absorb and store energy from food, while Bacteroidetes do the opposite. This study showed that sepsis changed the gut microbiota, in which the number of Firmicutes decreased and the number of Bacteroidetes increased, which led to a significant decrease in food intake and energy storage in the rats. Therefore, the imbalance in the gut microbiota further aggravates the lung injury induced by sepsis. After ADMSC intervention in the SD rats, the proportion of Bacteroidetes and Firmicutes was partially corrected, energy intake and consumption was gradually balanced, the mortality of sepsis was effectively reduced, the inflammatory response in rats with sepsis was reduced, and the sepsis-induced ALI was improved.

Therefore, ADMSCs may reduce lung injury by blocking the release of inflammatory factors, or it may improve the ALI caused by sepsis by normalizing the intestinal flora structure of septic rats, increasing beneficial bacteria, and reducing harmful bacteria. Overall, we studied the therapeutic effect of ADMSCs on ALI in sepsis and found that treatment with ADMSCs may improve ALI and reduce systemic inflammation through alterations to the gut microbiota, so as to significantly improve the survival rate of rats with sepsis. A shortcoming of this study is that it is not yet verified whether ADMSCs may damage other organs in rats with sepsis at the same time, or whether they have a tendency to aggregate. This paper may provide potential drug treatment options for patients with sepsis, that is, to maintain the balance of the body's own response by controlling the inflammatory response and maintaining the stability of the intestinal microecology.

\section{Conclusions}

This is the first basic study on the changes of intestinal microorganisms caused by ADMSCs in the treatment of septic rats with ALI. The results show that ADMSCs may improve the ALI induced by CLP by 
regulating intestinal microflora, providing a potential mechanism for MSCs to treat sepsis.

\section{Abbreviations}

ADMSCs: Adipose-derived mesenchymal stem cells; ALI: Acute lung injury; H\&E: hematoxylin and eosin; ELISA: enzyme-linked immunosorbent assay; PCoA: Principal coordinates analysis; MSCs: Mesenchymal stem cells; OTUs: Operational taxonomic units.

\section{Declarations}

Authors' contributions

All the authors contributed substantially to the work presented in this article. TWS, JYS, and XFD conceived the study. JYS and XFD contributed to the study protocol and wrote the article. XGD, HYL and TWS revised the article. The corresponding author had full access to all of the data and the final responsibility for the decision to submit this article for publication. All authors read and approved the final manuscript.

Ethics approval and consent to participate

Not applicable.

Consent for publication

Not applicable.

Availability of data and materials

The datasets used and/or analysed in the present study are available from the corresponding author on reasonable request.

Consent to participate

Not applicable.

Conflicts of interests

The authors have no conflicts of interest to disclose.

\section{References}

1. Singer M, Deutschman CS, Seymour CW, et al. The Third International Consensus Definitions for Sepsis and Septic Shock (Sepsis-3)[J]. Jama. 2016;315(8):801-10.

2. Annane D, Bellissant E, Cavaillon JM. Septic shock[J]. Lancet. 2005;365(9453):63-78. 
3. Haak BW, Wiersinga WJ. The role of the gut microbiota in sepsis[J]. Lancet Gastroenterol Hepatol. 2017;2(2):135-43.

4. Vincent JL, Marshall JC, Namendys-Silva SA, et al. Assessment of the worldwide burden of critical illness: the intensive care over nations (ICON) audit[J]. Lancet Respir Med. 2014;2(5):380-6.

5. Kwon WY, Suh GJ, Kim KS, et al. Niacin and Selenium Attenuate Sepsis-Induced Lung Injury by UpRegulating Nuclear Factor Erythroid 2-Related Factor 2 Signaling[J]. Crit Care Med. 2016;44(6):e37082.

6. Potz BA, Sellke FW, Abid MR. Endothelial ROS and Impaired Myocardial Oxygen Consumption in Sepsis-induced Cardiac Dysfunction[J]. J Intensive Crit Care, 2016, 2(1).

7. Schortgen F, Asfar P. Update in sepsis and acute kidney injury 2014[J]. Am J Respir Crit Care Med. 2015;191(11):1226-31.

8. Zhao L, An R, Yang Y, et al. Melatonin alleviates brain injury in mice subjected to cecal ligation and puncture via attenuating inflammation, apoptosis, and oxidative stress: the role of SIRT1 signaling[J]. J Pineal Res. 2015;59(2):230-9.

9. Abraham E, Singer M. Mechanisms of sepsis-induced organ dysfunction[J]. Crit Care Med. 2007;35(10):2408-16.

10. Fan YW, Jiang SW, Chen JM, et al. A pulmonary source of infection in patients with sepsisassociated acute kidney injury leads to a worse outcome and poor recovery of kidney function[J]. World J Emerg Med. 2020;11(1):18-26.

11. Zhu LL, Zhou Q. Optimal infusion rate in antimicrobial therapy explosion of evidence in the last five years[J]. Infect Drug Resist. 2018;11:1105-17.

12. Hebbar K, Rigby MR, Felner El, et al. Neuroendocrine dysfunction in pediatric critical illness[J]. Pediatr Crit Care Med. 2009;10(1):35-40.

13. Irusen E, Matthews JG, Takahashi A, et al. p38 Mitogen-activated protein kinase-induced glucocorticoid receptor phosphorylation reduces its activity: role in steroid-insensitive asthma[J]. $J$ Allergy Clin Immunol. 2002;109(4):649-57.

14. Maumus M, Guerit D, Toupet $K$, et al. Mesenchymal stem cell-based therapies in regenerative medicine: applications in rheumatology[J]. Stem Cell Res Ther. 2011;2(2):14.

15. Zhu F, Guo GH, Chen W, et al. Effects of bone marrow-derived mesenchymal stem cells engraftment on vascular endothelial cell growth factor in lung tissue and plasma at early stage of smoke inhalation injury[J]. World J Emerg Med. 2010;1(3):224-8.

16. Sun $\mathrm{CK}$, Yen $\mathrm{CH}$, Lin YC, et al. Autologous transplantation of adipose-derived mesenchymal stem cells markedly reduced acute ischemia-reperfusion lung injury in a rodent model[J]. J Transl Med. 2011;9:118.

17. Le Blanc K, Tammik L, Sundberg B, et al. Mesenchymal stem cells inhibit and stimulate mixed lymphocyte cultures and mitogenic responses independently of the major histocompatibility complex[J]. Scand J Immunol. 2003;57(1):11-20. 
18. Gonzalez-Rey E, Anderson P, Gonzalez MA, et al. Human adult stem cells derived from adipose tissue protect against experimental colitis and sepsis[J]. Gut. 2009;58(7):929-39.

19. Gonzalez MA, Gonzalez-Rey E, Rico L, et al. Adipose-derived mesenchymal stem cells alleviate experimental colitis by inhibiting inflammatory and autoimmune responses[J]. Gastroenterology. 2009;136(3):978-89.

20. Nemeth K, Leelahavanichkul A, Yuen PS, et al. Bone marrow stromal cells attenuate sepsis via prostaglandin $E(2)$-dependent reprogramming of host macrophages to increase their interleukin-10 production[J]. Nat Med. 2009;15(1):42-9.

21. Ikarashi S, Tsuchiya A, Kawata Y, et al. Effects of Human Adipose Tissue-Derived and Umbilical Cord Tissue-Derived Mesenchymal Stem Cells in a Dextran Sulfate Sodium-Induced Mouse Model[J]. Biores Open Access. 2019;8(1):185-99.

22. Taeb AM, Hooper MH, Marik PE. Sepsis. Current Definition, Pathophysiology, Diagnosis, and Management[J]. Nutr Clin Pract. 2017;32(3):296-308.

23. Li D, Wang P, Wang P, et al. The gut microbiota: A treasure for human health[J]. Biotechnol Adv. 2016;34(7):1210-24.

24. Hooper LV, Macpherson AJ. Immune adaptations that maintain homeostasis with the intestinal microbiota[J]. Nat Rev Immunol. 2010;10(3):159-69.

25. Dorman G, Kocsis-Szommer K, Spadoni C, et al. MMP inhibitors in cardiac diseases: an update[J]. Recent Pat Cardiovasc Drug Discov. 2007;2(3):186-94.

26. Luo JX, Zhang Y, Hu XY. The Effect of Modified Sini Decoction on Survival Rates of Patients with Hepatitis B Virus Related Acute-on-Chronic Liver Failure[J]. 2019, 2019: 2501847.

27. Stewart CJ, Embleton ND, Marrs ECL, et al. Longitudinal development of the gut microbiome and metabolome in preterm neonates with late onset sepsis and healthy controls[J]. Microbiome. 2017;5(1):75.

28. Haak BW, Prescott HC, Wiersinga WJ. Therapeutic Potential of the Gut Microbiota in the Prevention and Treatment of Sepsis[J]. Front Immunol. 2018;9:2042.

29. West CE, Renz H, Jenmalm MC, et al. The gut microbiota and inflammatory noncommunicable diseases: associations and potentials for gut microbiota therapies[J]. J Allergy Clin Immunol. 2015;135(1):3-13. quiz 14.

30. Klingensmith NJ, Coopersmith CM. The Gut as the Motor of Multiple Organ Dysfunction in Critical Illness[J]. Crit Care Clin. 2016;32(2):203-12.

31. Yoshihisa A. Altered Gut Flora and Gut Microbiome-Derived Metabolites in Heart Failure Patients in the Compensated and Decompensated Phases[J]. Circ J. 2018;83(1):30-1.

32. Ojima M, Motooka D, Shimizu K, et al. Metagenomic Analysis Reveals Dynamic Changes of Whole Gut Microbiota in the Acute Phase of Intensive Care Unit Patients[J]. Dig Dis Sci. 2016;61(6):162834. 
33. Rittirsch D, Huber-Lang MS, Flierl MA, et al. Immunodesign of experimental sepsis by cecal ligation and puncture[J]. Nat Protoc. 2009;4(1):31-6.

34. Zhao T, Pan B, Alam HB, et al. Protective effect of Cl-amidine against CLP-induced lethal septic shock in mice[J]. Sci Rep. 2016;6:36696.

35. Ding XF, Sun M, Guan FX, et al. Prenatal Exposure to LPS Alters The Intrarenal RAS in Offspring, Which Is Ameliorated by Adipose Tiss ue-Derived Mesenchymal Stem Cells[J]. Am J Hypertens. 2017;30(12):1211-9.

36. Logue JB, Stedmon CA, Kellerman AM, et al. Experimental insights into the importance of aquatic bacterial community composition to the degradation of dissolved organic matter[J]. 2016, 10(3): 533-545.

37. Walters W, Hyde ER, Berg-Lyons D, et al. Improved Bacterial 16S rRNA Gene (V4 and V4-5) and Fungal Internal Transcribed Spacer Marker Gene Primers for Microbial Community Surveys[J]. mSystems, 2016, 1(1).

38. Takai K, Horikoshi K. Rapid detection and quantification of members of the archaeal community by quantitative PCR using fluorogenic probes[J]. Appl Environ Microbiol. 2000;66(11):5066-72.

39. Xie J, Liu Y, Chen B, et al. Ganoderma lucidum polysaccharide improves rat DSS-induced colitis by altering cecal microbiota and gene expression of colonic epithelial cells[J]. Food Nutr Res, 2019, 63.

40. Vega-Magana N, Delgado-Rizo V, Garcia-Benavides L, et al. Bacterial Translocation Is Linked to Increased Intestinal IFN-gamma, IL-4, IL-17, and mucin-2 in Cholestatic Rats[J]. Ann Hepatol. 2018;17(2):318-29.

41. Shogan BD, Smith DP, Christley S, et al. Intestinal anastomotic injury alters spatially defined microbiome composition and function[J]. Microbiome. 2014;2:35.

42. Alam A, Leoni G, Quiros $M$, et al. The microenvironment of injured murine gut elicits a local prorestitutive microbiota[J]. 2016, 1: 15021.

43. Berry D, Stecher B, Schintlmeister A, et al. Host-compound foraging by intestinal microbiota revealed by single-cell stable isotope probing[J]. Proc Natl Acad Sci U S A. 2013;110(12):4720-5.

44. Zhang Z, Wu X, Cao S, et al. Chlorogenic Acid Ameliorates Experimental Colitis by Promoting Growth of Akkermansia in Mice[J]. Nutrients, 2017, 9(7).

45. Hu L, Yin C, Zhao F, et al. Mesenchymal Stem Cells: Cell Fate Decision to Osteoblast or Adipocyte and Application in Osteoporosis Treatment[J]. 2018, 19(2).

46. Lin GL, McGinley JP, Drysdale SB, et al. Epidemiology and Immune Pathogenesis of Viral Sepsis[J]. Front Immunol. 2018;9:2147.

47. Lyons JD, Coopersmith CM. Pathophysiology of the Gut and the Microbiome in the Host Response[J]. Pediatr Crit Care Med. 2017;18(3_suppl Suppl 1):46-s49.

48. Rainer F, Horvath A, Sandahl TD, et al. Soluble CD163 and soluble mannose receptor predict survival and decompensation in patients with liver cirrhosis, and correlate with gut permeability and bacterial translocation[J]. Aliment Pharmacol Ther. 2018;47(5):657-64. 
49. Ding XF, Liang HY, Sun JY, et al. Adipose-derived mesenchymal stem cells ameliorate the inflammatory reaction in CLP-induced septic acute lung injury rats via sTNFR1[J]. 2019.

50. Liang H, Ding X, Yu Y, et al. Adipose-derived mesenchymal stem cells ameliorate acute liver injury in rat model of CLP induced-sepsis via sTNFR1[J]. Exp Cell Res. 2019;383(1):111465.

51. Pan $\mathrm{H}$, Guo R, Zhu J, et al. A gene catalogue of the Sprague-Dawley rat gut metagenome[J]. Gigascience, 2018, 7(5).

\section{Supplementary Figure Legend}

Figure S1. Macroscopic view of the rat model.

\section{Figures}



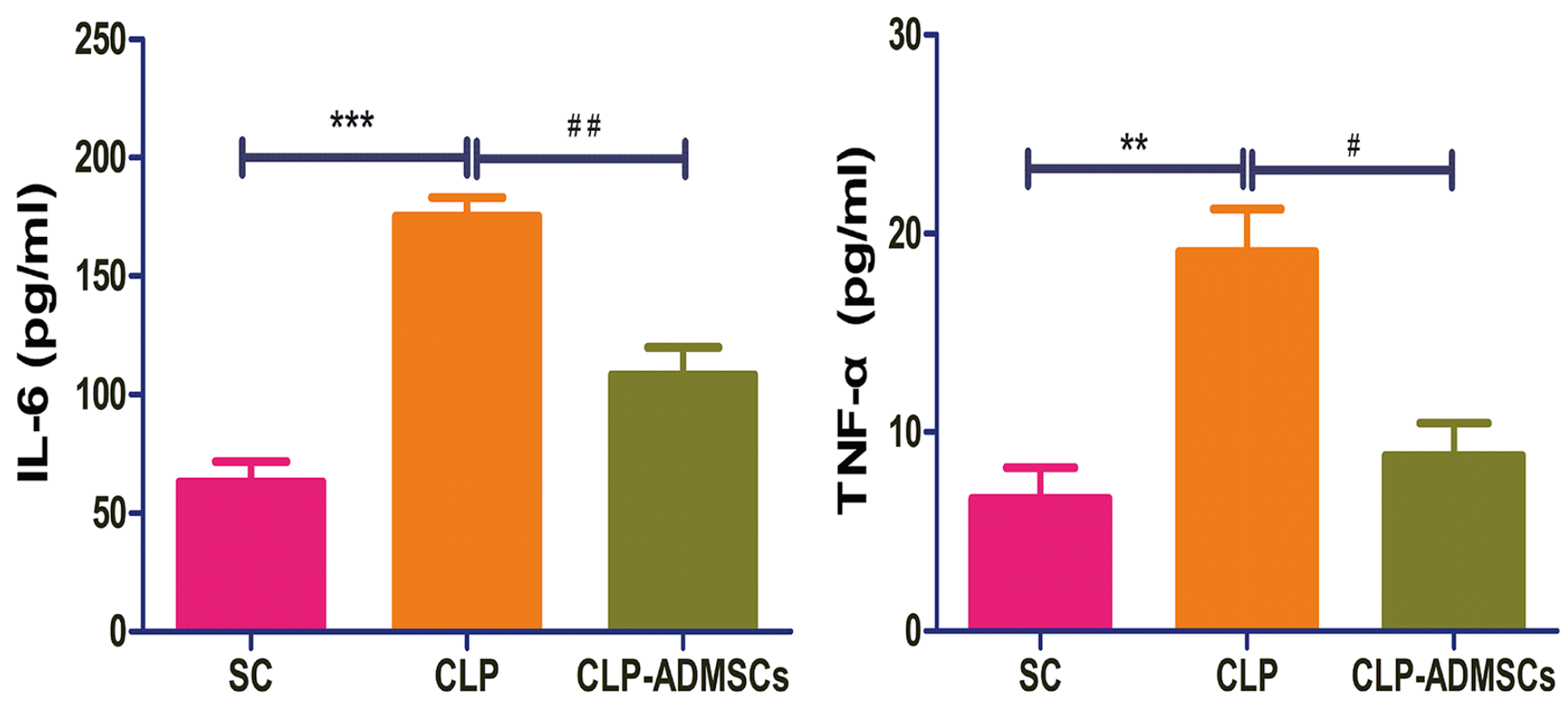

SC

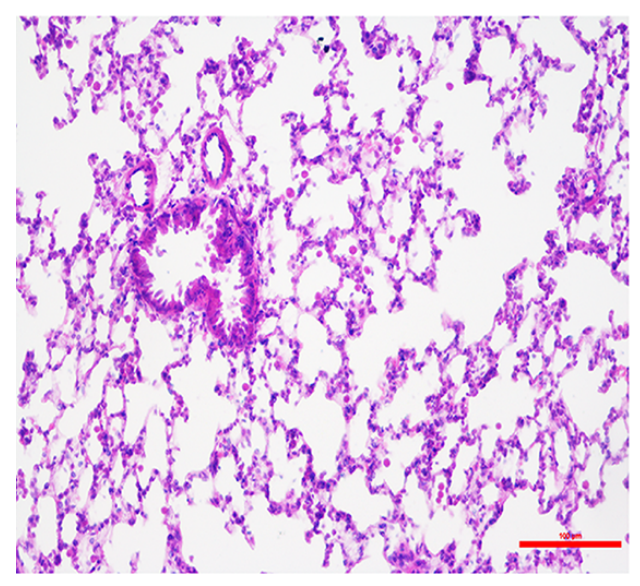

\section{C}

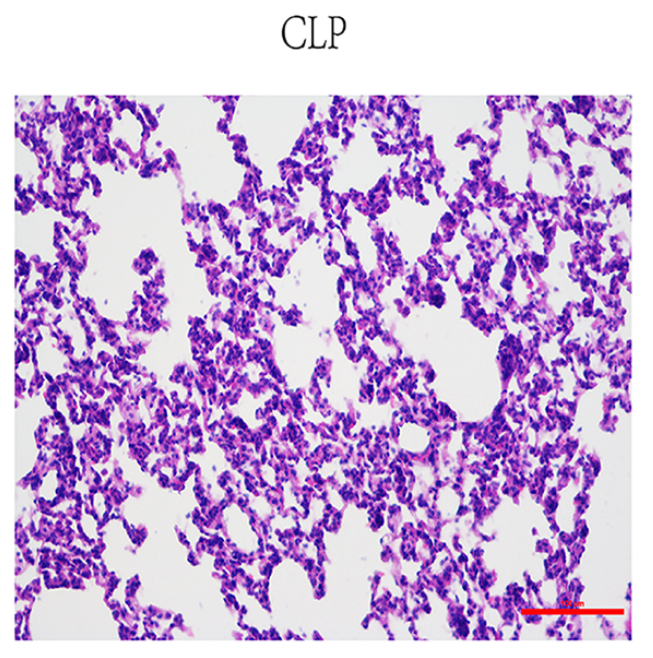

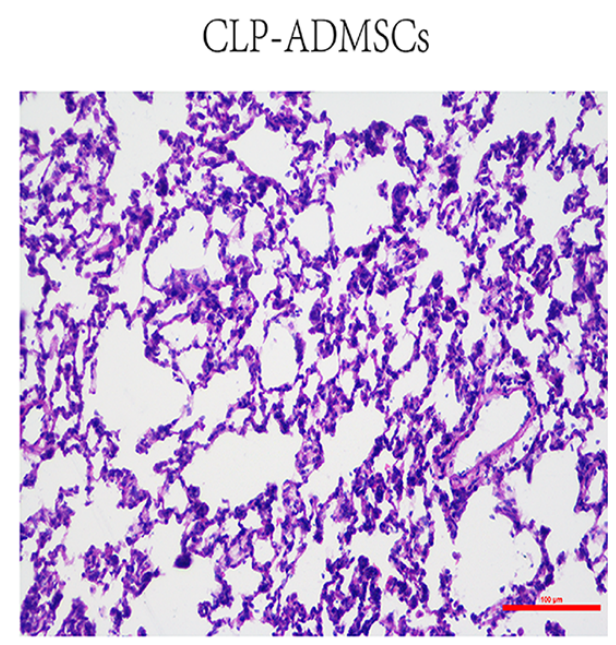

Figure 1

Effect of adipose-derived mesenchymal stem cells (ADMSCs) on systemic inflammatory response in rats with cecal ligation and puncture (CLP)-induced acute lung injury (ALI). The serum levels of IL-6 (A) and TNF-a (B) were measured using enzyme-linked immunosorbent assay (ELISA). ${ }^{*}$ Compared with the sham control (SC) group; \#Compared with the CLP group; * \#, P< 0.05; **, \#\#, P< 0.01; ***, \#\#\#, P< 0.001 . (C) Effect of CLP and ADMSCs on 24-hour lung histology. Hematoxylin and eosin staining showed that inflammatory infiltration, edema, and hemorrhage of rat lung tissue from the CLP group were significantly enhanced. Treatment with ADMSCs improved these anomalies (bar $=100 \mu \mathrm{m})$. 


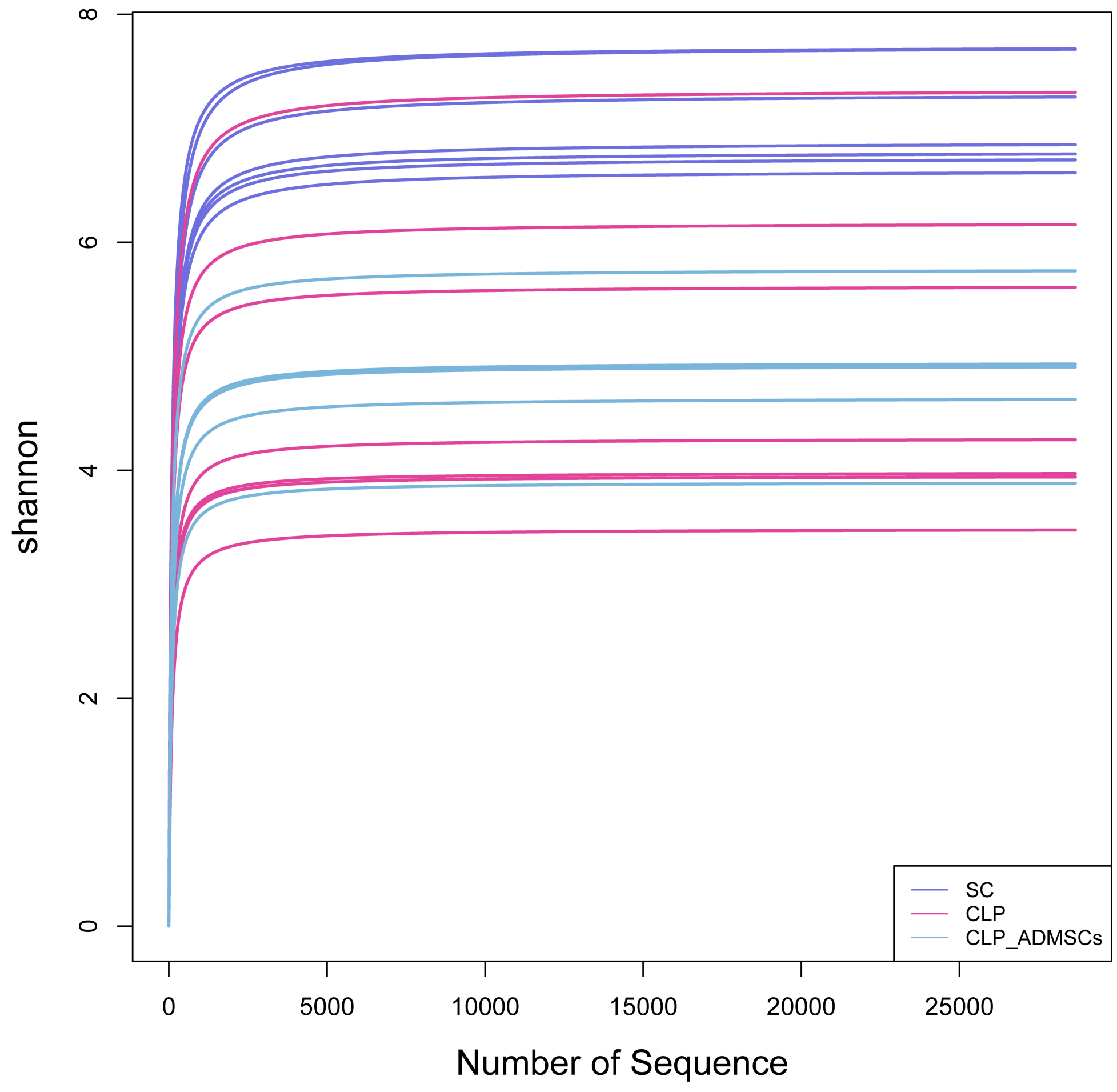

Figure 2

Abundance analysis of intestinal microecology. The abscissa represents the number of randomly selected sequences. The ordinate represents the number of operational taxonomic units (OTUs) observed. 


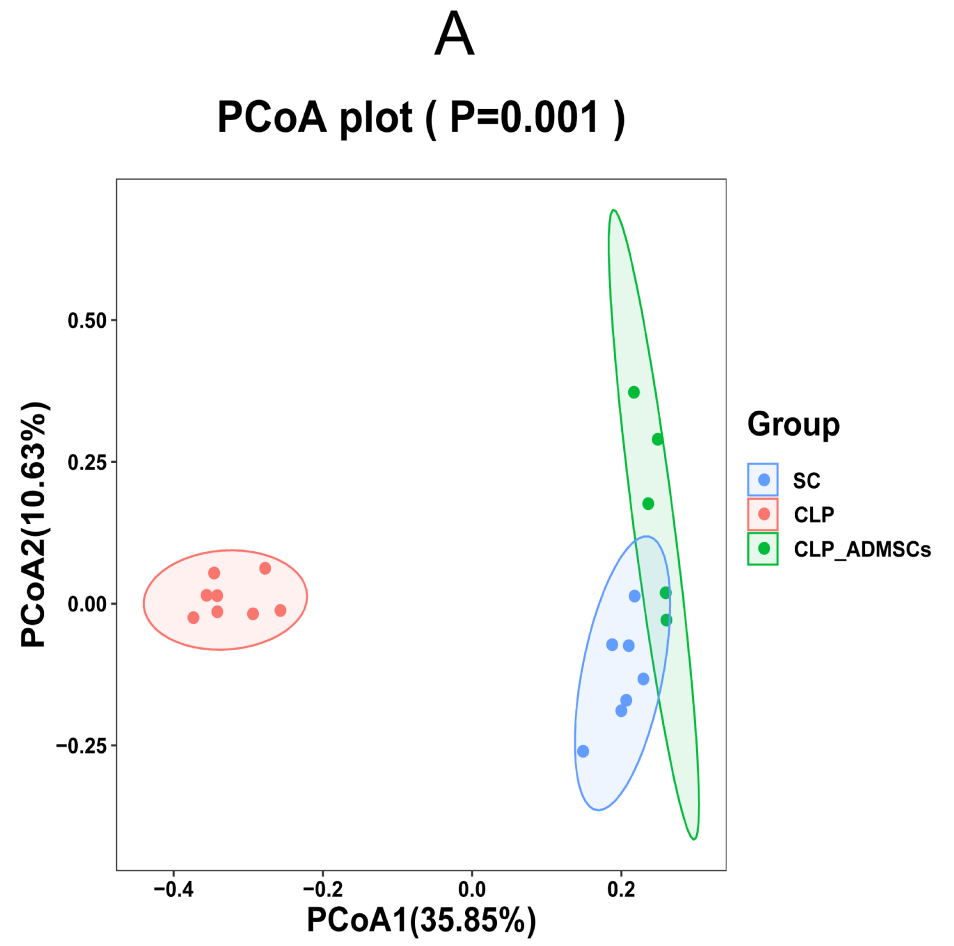

B

Principal Component Analysis ( $P=0.001)$

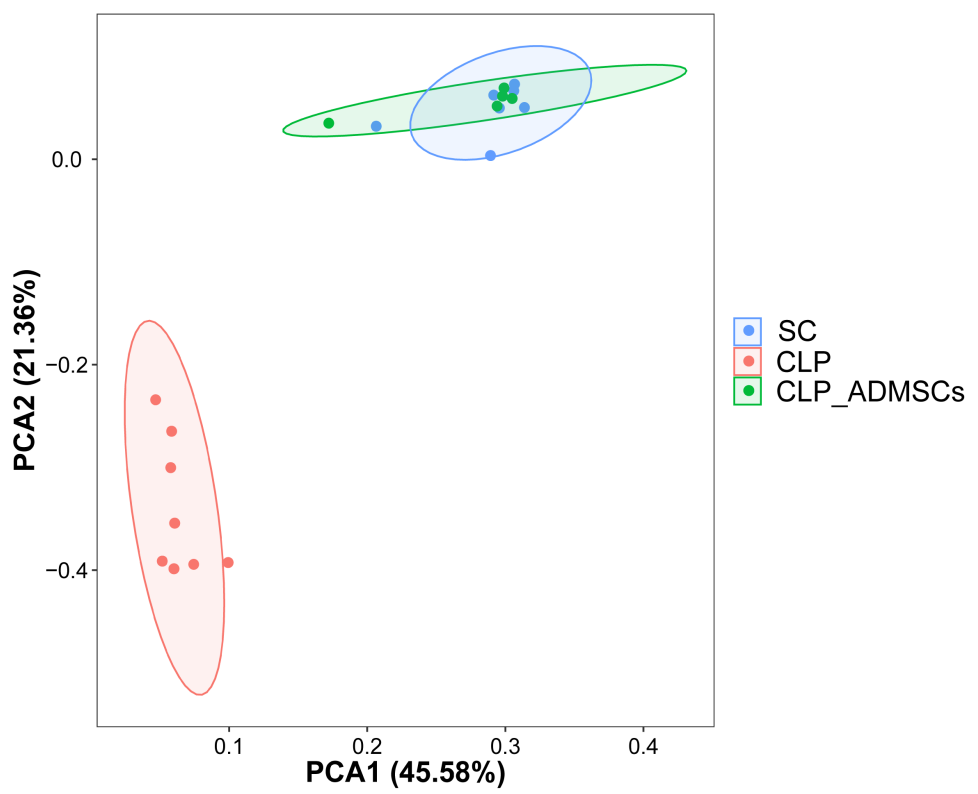

Figure 3

(A) Principal coordinates analysis (PCoA) based on unweighted UniFrac values of rats from the different treatment groups. (B) Principal component analysis (PCA) of rats from the different treatment groups. 
A

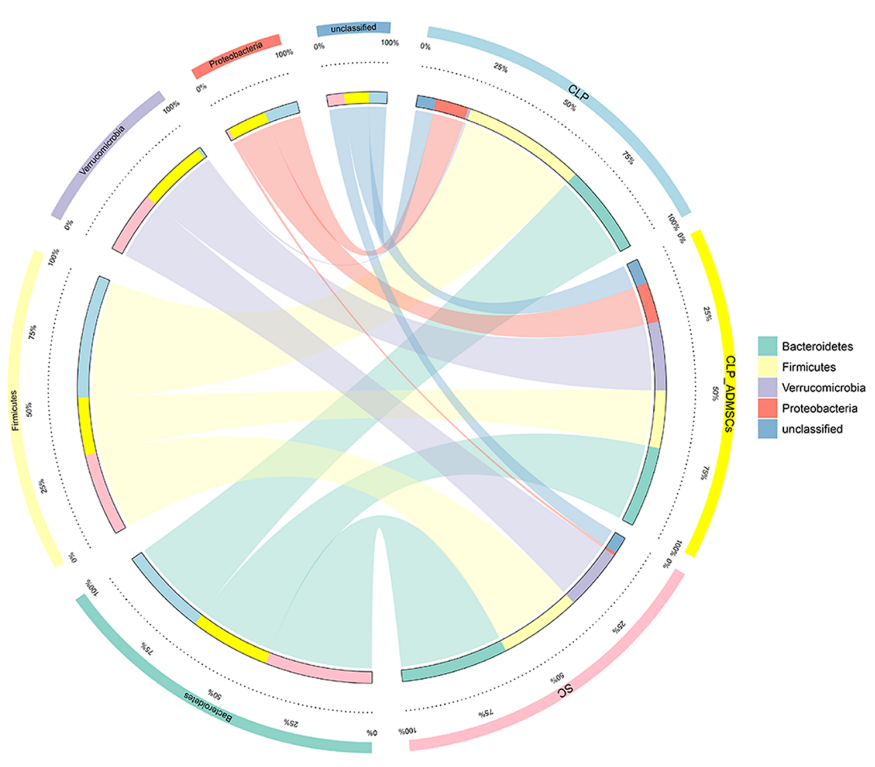

C

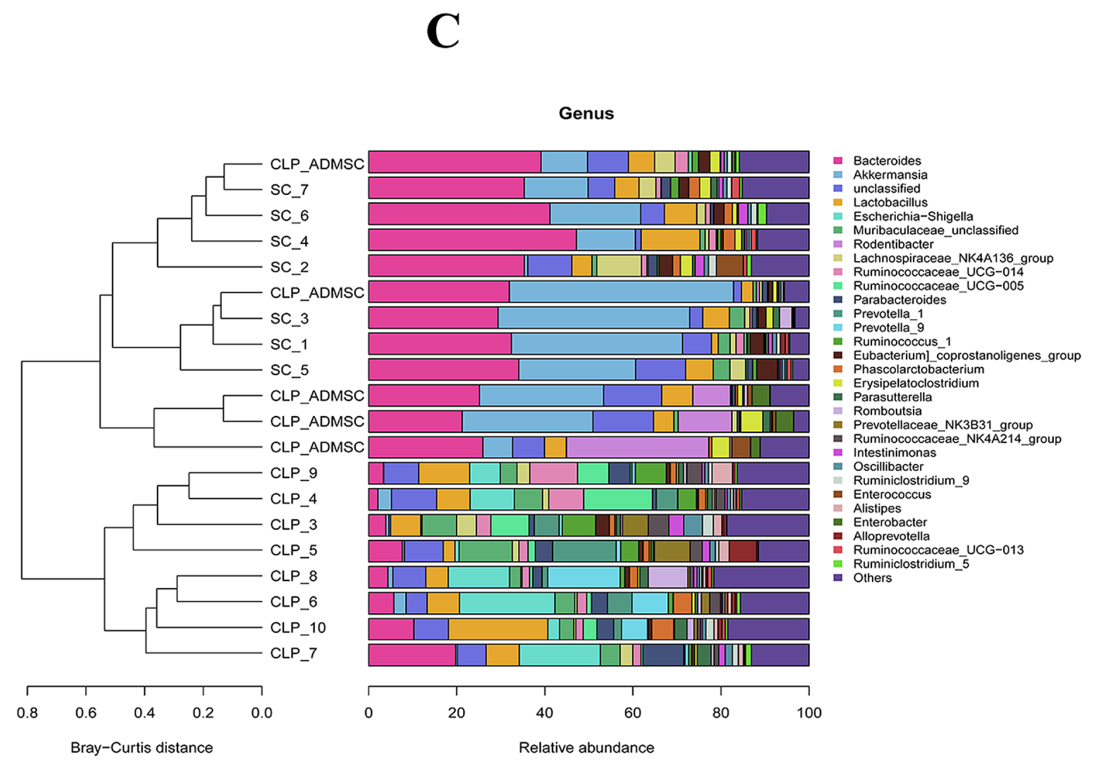

B

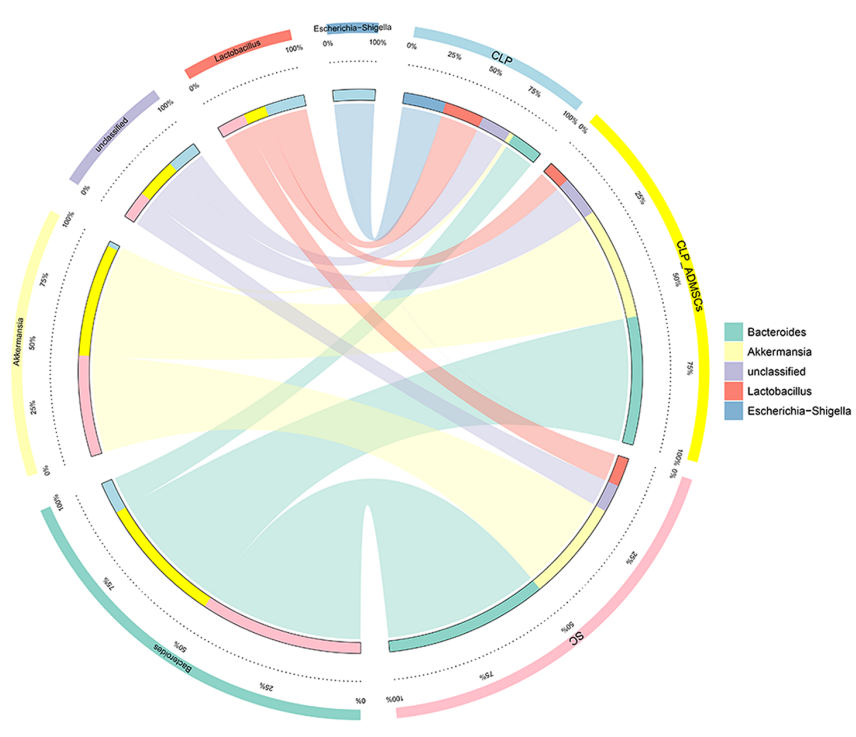

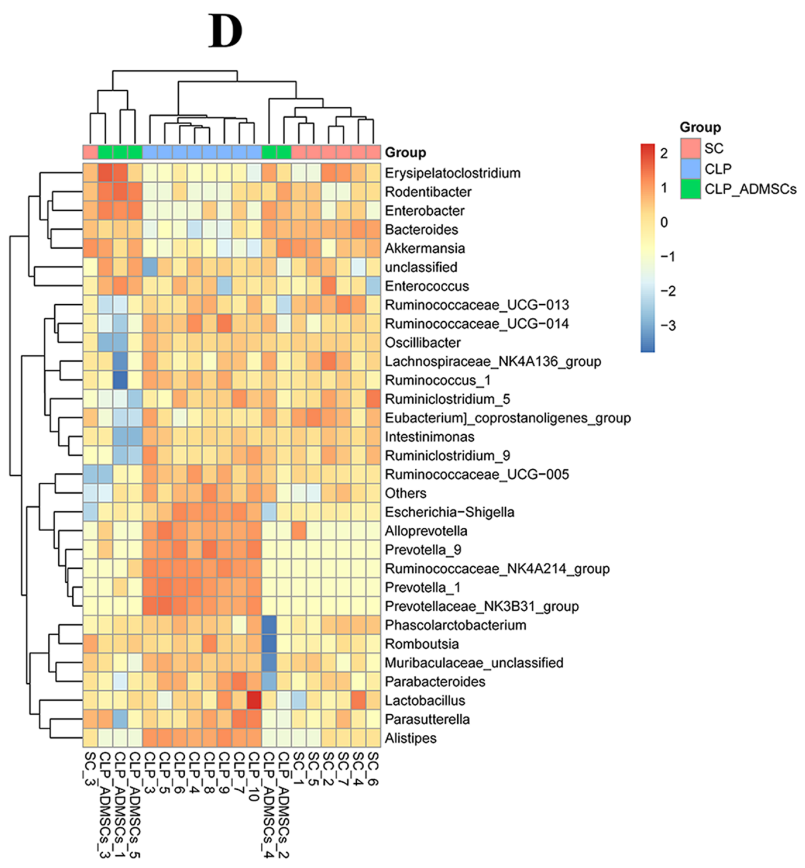

\section{Figure 4}

(A) Distribution of phylum in the gut microbiota of rats from the different treatment groups. (B) Distribution of genus in the gut microbiota of rats from the different treatment groups. (C) Cluster graphs. Left: The Bray-Curtis distance clustering tree structure. Closer sample clustering and shorter branches indicate a higher degree of similarity in the sample species composition. Right: The relative abundance distribution map of each sample at the gate level. A larger proportion indicates higher abundance. (D) Heat map. The gradient colors from blue to red reflect the change in abundance from low to high. Colors closer to blue indicate a lower abundance; colors closer to red indicate a higher abundance. 